\begin{tabular}{|c|l|}
\hline Title & Three dimensional observation of an immiscible polymer blend subjected to a step electric field under shear flow \\
\hline Author(s) & Orihara, Hiroshi; Nishimoto, Y uki; A ida, Kohei; Na, Y ang Ho \\
\hline Citation & $\begin{array}{l}\text { Physical Review E, 83(2), 026302 } \\
\text { https://doi.org/10.1103/PhysRevE.83.026302 }\end{array}$ \\
\hline Issue Date & 2011-02 \\
\hline Doc URL & http://hdl.handle.net/2115/45021 \\
\hline Rights & O2011 A merican Physical Society \\
\hline Type & article \\
\hline File Information & PRE832_026302.pdf \\
\hline
\end{tabular}

Instructions for use 


\title{
Three-dimensional observation of an immiscible polymer blend subjected to a step electric field under shear flow
}

\author{
Hiroshi Orihara, ${ }^{*}$ Yuki Nishimoto, Kohei Aida, and Yang Ho Na \\ Division of Applied Physics, Faculty of Engineering, Hokkaido University, Sapporo, Hokkaido 060-8628, Japan
}

(Received 1 November 2010; published 7 February 2011)

\begin{abstract}
We have investigated the structural change of an immiscible blend consisting of two polymers with equal viscosity subjected to a step electric field under shear flow. During the process, three-dimensional images were successfully acquired with a confocal scanning laser microscope, and at the same time, the transient shear stress was also measured. From the images, the interface tensor was calculated. In a blend of polymers with equal viscosity, the total shear stress may consist of the viscous, interfacial, and electric stresses. An experiment was performed to separate these stresses, and the results are discussed in terms of the interface tensor.
\end{abstract}

DOI: 10.1103/PhysRevE.83.026302

PACS number(s): 47.65.Gx, 83.80.Tc, 47.57.Bc

\section{INTRODUCTION}

Immiscible fluid blends (emulsions) exhibit many interesting rheological characteristics, which are closely related to morphological changes. When subjected to a flow, such blends undergo changes through complex processes such as deformation, breakup, and coalescence. Tucker and Moldenaers [1] reviewed the recent successes in understanding these relationships through theory and experimentation. In 1991, Doi and Ohta [2] developed a semiphenomenological theory in terms of the interface tensor:

$$
q_{\alpha \beta}=\frac{1}{V} \int_{S}\left(n_{\alpha} n_{\beta}-\frac{1}{3} \delta_{\alpha \beta}\right) d S,
$$

where $\mathbf{n}$ is the unit vector normal to the interface, $V$ is the system volume, and the integration is performed over the whole interface area $S$ of the system. The excess stress due to the interfacial tension is expressed as $-\Gamma q_{\alpha \beta}$ [3-5], where $\Gamma$ is the interfacial tension. Recently, Takahashi et al. [6] conducted a detailed experimental evaluation. They observed the shape change of droplets after applying a large step strain using a specially designed apparatus. The shape was classified into three types: ellipsoid, rod-like shape, and dumbbell, and the corresponding surface tensors were calculated. The result was compared with the shear stress measured separately with a rheometer, and Eq. (1) was shown to hold. However, when the viscosities of two fluids are different, there is an additional contribution to the total shear stress due to the velocity field at the surface and the viscosity difference between the fluids $[3,7,8]$.

In addition to the flow, an electric field can bring about morphological changes in blends. In fact, for two fluids with mismatched electric properties, such as permittivity and conductivity, under the application of an electric field, remarkable changes have been reported [9-13]. The morphological change is accompanied by a viscosity change. This phenomenon is called the electrorheological (ER) effect. In the absence of an electric field, droplets of one fluid are dispersed within the matrix of the other. When subjected to a step electric field, on the other hand, the droplets become elongated along the

*orihara@eng.hokudai.ac.jp field due to the Maxwell stress exerted on the interfaces, and occasionally a network structure is formed, resulting in an apparent increase in viscosity [14-16]. The transient response of shear stress to a step electric field has been investigated in detail and scaling properties have been found [17]. For example, when ER blends are subjected to a step electric field at a constant shear rate, the transient apparent viscosity depends only on strain, as long as $\dot{\gamma} / E^{2}$ is constant, where $\dot{\gamma}$ and $E$ are the shear rate and electric field, respectively.

Quite recently, we investigated the relationship between the structure and the shear stress of an immiscible blend subjected to a step electric field [18]. Because we used a blend consisting of two fluids with different viscosities, however, the obtained results were unclear due to the effect related to the viscosity difference. For an immiscible blend with equal viscosity, on the other hand, the total shear stress can be separated into the viscous stress and the interfacial stress. The former is expressed as $\eta \dot{\gamma}$, where $\eta$ is the viscosity, and the latter as $-\Gamma q_{z x}$, under the assumption that the flow velocity and the velocity gradient are along the $x$ and $z$ axes, respectively. Under an electric field, another contribution from the Maxwell stress may also appear, which will be called the electric stress hereafter. In this study, an immiscible blend with equal viscosity was used and the three contributions were experimentally separated in the transient process after a step electric field was applied under a steady shear flow. At the same time, three-dimensional (3D) structures were observed during the process with a new system combining a confocal laser scanning microscope (CLSM) and a rheometer. The interface tensor is calculated, and we show that not only the interfacial stress, but also the electric stress, can be well described by the surface tensor.

\section{EXPERIMENTS}

In the experiment, we used two polymers. One was a liquid-crystalline polymer (LCP) (Fig. 1) [10]. The other was a polyisobutylene (PIB), which was a mixture of two PIB's with different molecular weights of 1350 and 500. The two PIB's were mixed so that the viscosity was equal to that of $\mathrm{LCP}$; the mixing ratio was $9(\mathrm{MW}=1350): 1(\mathrm{MW}=500)$. All experiments were conducted at $28^{\circ} \mathrm{C}$, where the LCP was in the isotropic phase, and so the mesogens play a role only in enhancing the permittivity and conductivity of the LCP. 


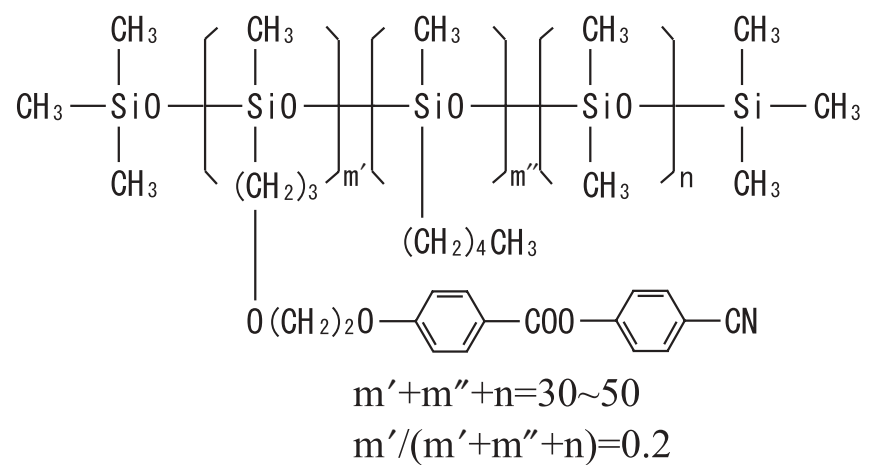

FIG. 1. Liquid-crystalline polymer.

At $28^{\circ} \mathrm{C}$, the viscosities of the LCP and PIB are the same, 33.5 Pa s. The relative dielectric constant and conductivity of the LCP are 16.0 and $4 \times 10^{-9} \Omega^{-1} \mathrm{~m}^{-1}$, and those of PIB are 2.7 and $3.3 \times 10^{-11} \Omega^{-1} \mathrm{~m}^{-1}$, respectively. We used PIB because its refractive index (1.490) matches well with that of the LCP (1.488). To distinguish between the LCP and PIB, a small amount of fluorescent dye, IANBD amide (Molecular Probes), was added to the LCP before mixing the two polymers. It was confirmed that the dye did not dissolve in PIB. The polymers were then blended at a volume ratio of LCP $:$ PIB $=1: 8$. For this blend, in the absence of an electric field, the LCP was dispersed in PIB as droplets.

A schematic picture of the experimental system is shown in Fig. 2(a). The sample was sandwiched between a bottom glass plate with an indium tin oxide (ITO)-coated electrode and the rotating plate of the rheometer (MCR301, Anton Paar). The diameter of the rotating plate was $35 \mathrm{~mm}$, and the gap between the plates was $200 \mu \mathrm{m}$. The shear rate was measured at the edge of the rotating disk, and the shear stress was calculated from the measured torque under the assumption of a Newtonian fluid because a parallel-plate rheometer was used in the experiment. Although immiscible polymer blends under an electric field are not generally Newtonian [19], the above assumption is not considered to seriously affect our conclusions in this paper because the contribution to the stress around the edge is dominant and we only roughly estimate the shear stress. Observations were made through the bottom glass plate with a CSLM (IX71, Olympus and CSU22, Yokogawa). We carried out both structural observation and rheological measurement at the same time, and the relationship between morphology and rheology is discussed on the basis of the results.

To construct 3D images, a piezoactuator (P-721.10, PI) was attached to the objective lens for vertical displacement, with the $x, y$, and $z$ axes taken as the flow, vorticity, and velocity gradient (the electric field) directions, respectively. A triangular voltage of $5 \mathrm{~Hz}$ was applied to the piezoactuator so that the objective lens or the focal plane oscillated vertically between 11 and $67 \mu \mathrm{m}$ above the bottom glass plate, as shown in Fig. 2(b) [20]. We used a water-immersion objective lens $(\times 40)$. The gap between the lens and the bottom glass plate was filled with water such that the actual height of the 3D images was about $56 \mu \mathrm{m}$. To construct each 3D image, 50 images were captured per half-period $(0.1 \mathrm{~s})$ at a frame rate of $500 \mathrm{fps}$. The image processing was done with Vox Blast (Vay Tek) and ImageJ. During the capture of one 3D image in this experiment, a shear rate of $1 \mathrm{~s}^{-1}$ brought about a total strain of $10 \%$. This was not negligible, and correction was made as described in the appendix.

Before each measurement, we applied a preshear of $1 \mathrm{~s}^{-1}$ for $3 \mathrm{~h}$ to ensure that the blend was in a steady state under the constant shear. We then applied a step ac electric field to the blend under steady shear flow by using a synthesizer (Multifunction Synthesizer 1940, NF Electric Instruments) and a high-voltage amplifier (609C-6, Trek). An ac electric field of $1000 \mathrm{~Hz}$ was used to avoid the conductivity effect.

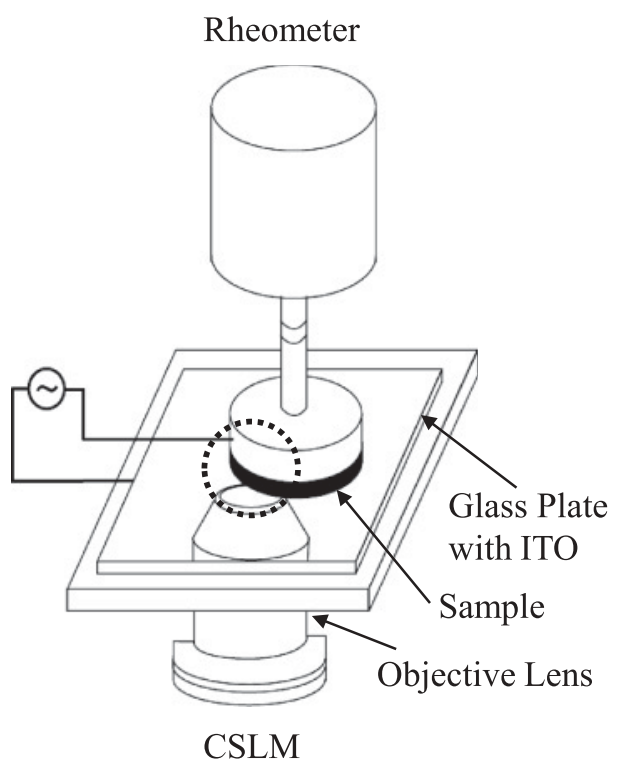

(a)

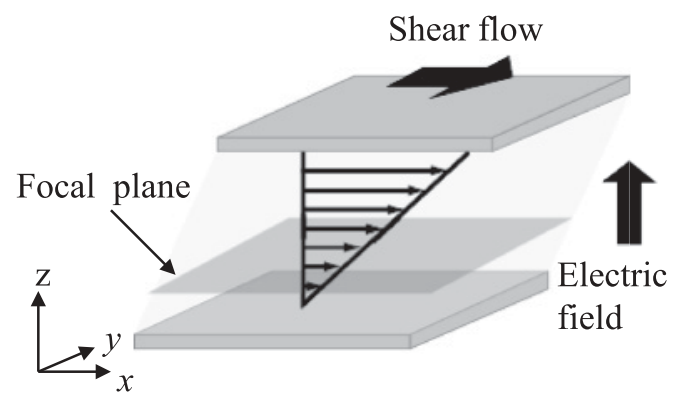

(b)

FIG. 2. (a) Schematic illustration of the system combining a rheometer and a CSLM, and (b) details of the sample space. 


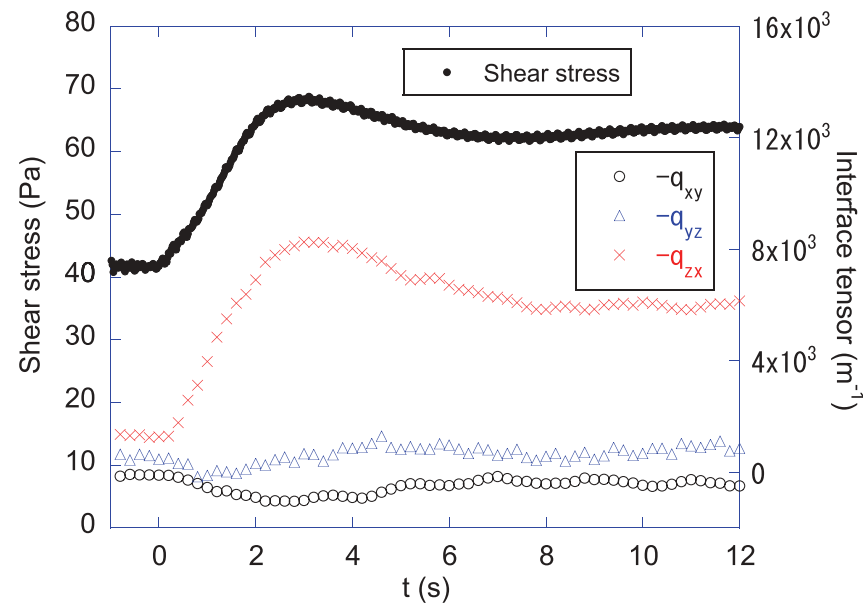

FIG. 3. (Color online) Transient shear stress under a step electric field. Off-diagonal elements of the interface tensor are also shown.

\section{RESULTS AND DISCUSSION}

Figure 3 shows the transient shear stress, when the blend was subjected to an ac electric field with an amplitude of $6 \mathrm{kV} / \mathrm{mm}$ at a constant shear rate of $1 \mathrm{~s}^{-1}$. The shear stress gradually increases after the electric field is applied at $t=0$. It passes through a local maximum and minimum and then gradually increases until reaching a steady value. The transient 3D images captured together with the stress measurement are shown in Fig. 4. The bright parts are a LCP, into which a small amount of fluorescent dye was added. In the absence of an electric field, the droplets of LCP are dispersed within the PIB matrix $(0 \mathrm{~s})$. Immediately after application of the step electric field, all the droplets are elongated along the electric field and some coalesce with one another to form columns (1 s). The same behavior has been observed without shear flow, and the elongation and coalescence are attributed to the Maxwell stress exerted on the interface and the induced dipole-dipole interaction between droplets, respectively [20]. Then, the columns gradually tilt toward the flow direction (2-3 s), causing the shear stress to increase. Further tilting may decrease the shear stress ( $4 \mathrm{~s})$. In the meantime, the columns become linked with each other to form a network structure (4-8 s).

Now, let us quantitatively investigate the structural change in terms of the interface tensor defined in Eq. (1). This tensor is symmetrical and traceless, and expresses the structural anisotropy. All the elements are zero in the case of a sphere, whereas the diagonal elements are nonzero for a general ellipsoid with semiaxes along the reference frame axes. If the lengths of the semiaxes are $a, b$, and $c$ (parallel to the $x, y$, and $z$ axes, respectively), note that $q_{x x}<q_{y y}<q_{z z}$ if $a>b>c$; in other words, the diagonal component along the elongated direction becomes small. Furthermore, when the ellipsoid tilts toward the $x$ direction, the off-diagonal elements, $q_{x z}=q_{z x}$, become nonzero.

Figure 5 shows the time dependence of the diagonal elements corresponding to the shear stress in Fig. 3. In the absence of an electric field, all the components are almost zero because droplets are nearly spherical. Immediately after the step electric field is applied, $q_{z z}$ decreases while both $q_{x x}$ and $q_{y y}$ increase, indicating that the droplets are elongated along the electric field (the $z$ axis). Next, $q_{x x}$ decreases and $q_{z z}$ increases. The time dependence of the off-diagonal elements $-q_{\alpha \beta}$ is plotted in Fig. 3. Both $-q_{x y}$ and $-q_{y z}$ are almost zero, as expected from the symmetry. On the other hand, $-q_{z x}$ increases immediately after the electric field is applied, passes through a maximum and minimum, and then gradually increases until reaching a steady value. This behavior is quite similar to that of the shear stress. In fact, as mentioned in Sec. I, the interfacial stress is expressed as $-\Gamma q_{\alpha \beta}$ [3-5]. However, it should be noted that the total shear stress consists of contributions other than the interfacial tension. We will attempt to experimentally separate the contributions
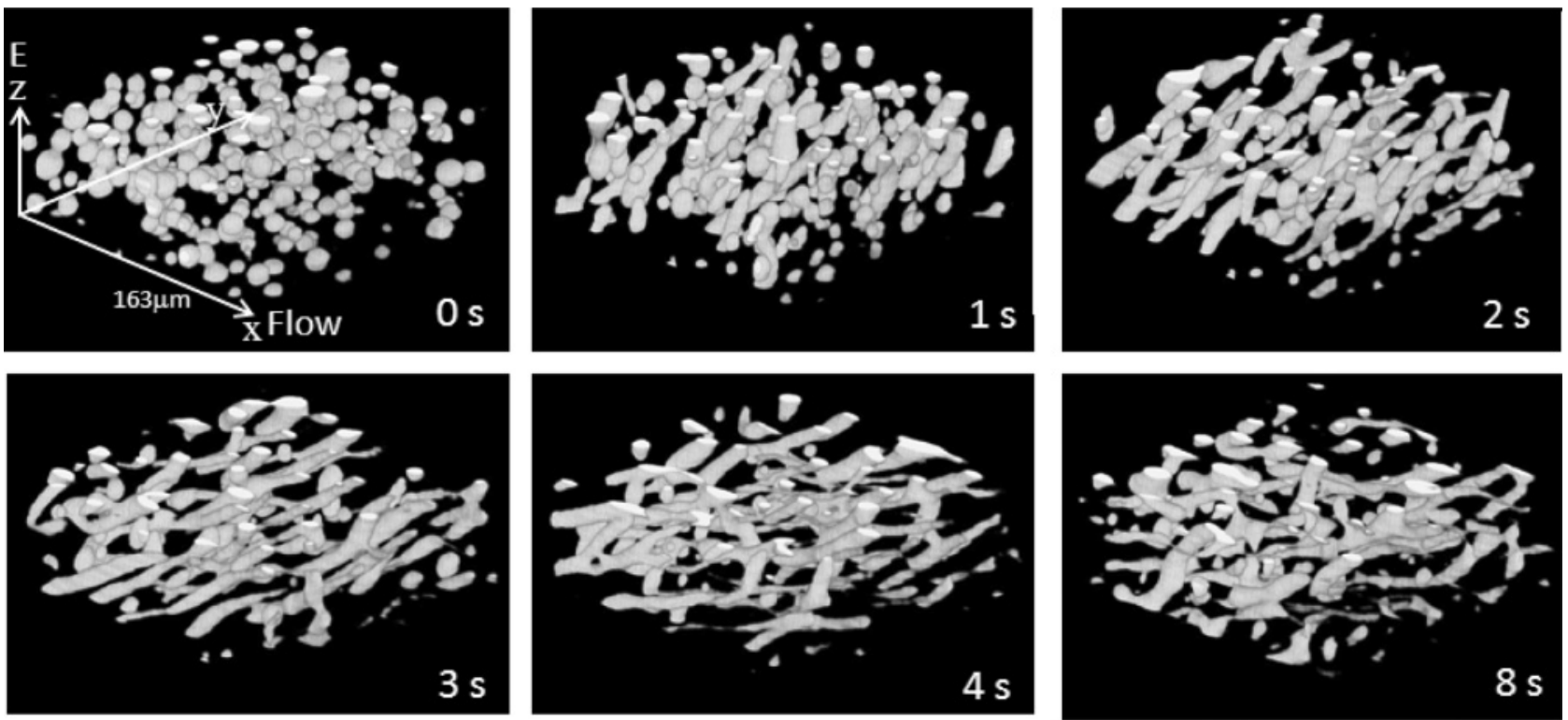

FIG. 4. 3D images in transient process, where the $x, y$, and $z$ axes are taken along the flow, vorticity, and velocity gradient (the electric field) directions, respectively. The corresponding movie is provided in Ref. [21]. 


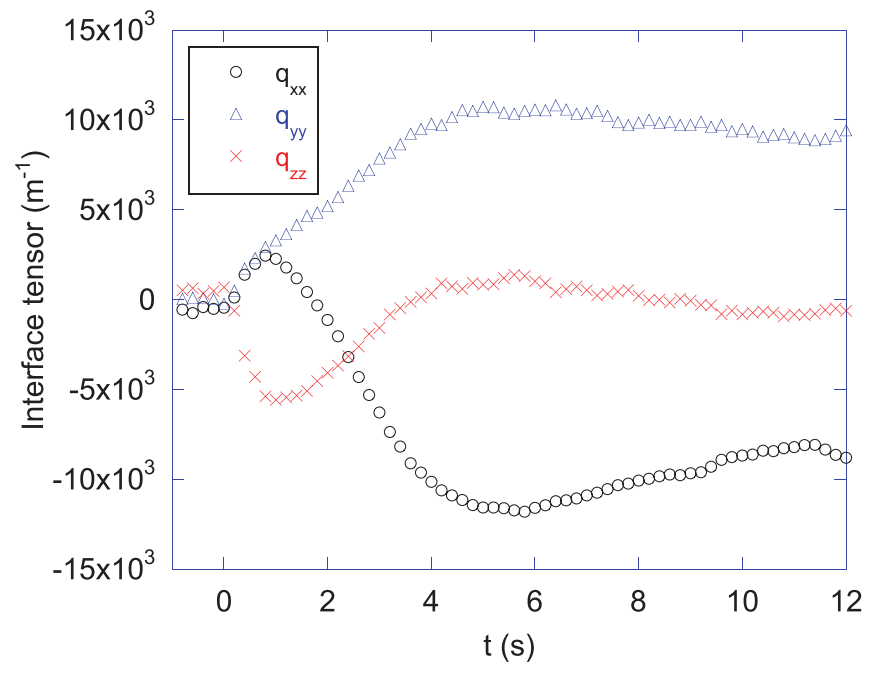

FIG. 5. (Color online) Time dependence of diagonal elements of the interface tensor.

later, but next let us investigate the structural anisotropy in more detail.

To visualize the interface tensor, we use an ellipsoid, which is assumed to be present in a volume $V_{e}$. The interface tensor for the ellipsoid of the principal axes parallel to the reference frame axes can be expressed as

$$
q_{\alpha \alpha}=Q_{\alpha \alpha}-\frac{1}{3} Q(\alpha=x, y, z),
$$

where

$$
Q=Q_{x x}+Q_{y y}+Q_{z z} .
$$

Here, $Q$ is the surface area density, and $Q_{x x}, Q_{y y}$, and $Q_{z z}$ are

$$
\begin{aligned}
Q_{x x} \equiv & \frac{1}{V_{e}} \int_{S} n_{x}^{2} d S=\frac{1}{V_{e}} \int_{0}^{\pi} d \theta \int_{0}^{2 \pi} d \phi \\
& \times \frac{a b c(\sin \theta \cos \phi / a)^{2}}{\sqrt{(\sin \theta \cos \phi / a)^{2}+(\sin \theta \sin \phi / a)^{2}+(\cos \theta / c)^{2}}}, \\
Q_{y y} \equiv & \frac{1}{V_{e}} \int_{S} n_{y}^{2} d S=\frac{1}{V_{e}} \int_{0}^{\pi} d \theta \int_{0}^{2 \pi} d \phi \\
& \times \frac{a b c(\sin \theta \sin \phi / a)^{2}}{\sqrt{(\sin \theta \cos \phi / a)^{2}+(\sin \theta \sin \phi / a)^{2}+(\cos \theta / c)^{2}}}, \\
Q_{z z} \equiv & \frac{1}{V_{e}} \int_{S} n_{z}^{2} d S=\frac{1}{V_{e}} \int_{0}^{\pi} d \theta \int_{0}^{2 \pi} d \phi \\
& \times \frac{a b c(\cos \theta / c)^{2}}{\sqrt{(\sin \theta \cos \phi / a)^{2}+(\sin \theta \sin \phi / a)^{2}+(\cos \theta / c)^{2}}},
\end{aligned}
$$

and the off-diagonal elements $q_{x y}=q_{y z}=q_{z x}=0$. For a tilting ellipsoid, the interface tensor can be obtained by rotating the preceding diagonal tensor. Inversely, the ellipsoid corresponding to a given interface tensor is uniquely determined. Thus, the interface tensor can be expressed by an ellipsoid.
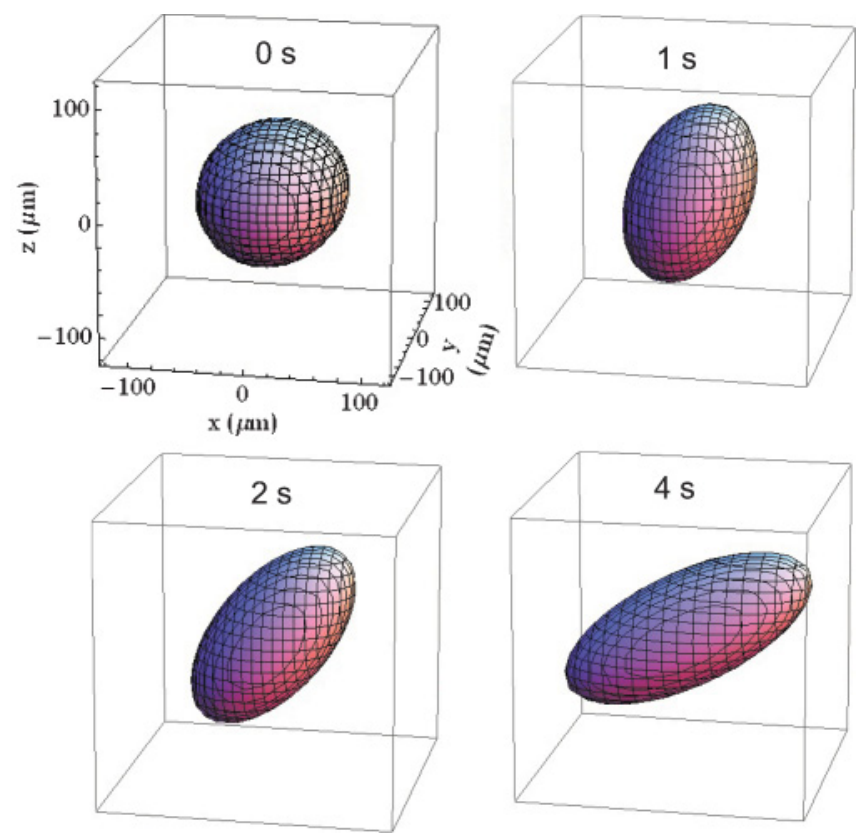

FIG. 6. (Color online) Time evolution of the ellipsoid corresponding to that of the surface tensor in Figs. 3 and 5. The corresponding movie is provided in Ref. [22].

For $V_{e}=100^{3} \mu \mathrm{m}^{3}$, the time evolution of the ellipsoid corresponding to Figs. 3 and 5 is shown in Fig. 6. In Fig. 6, the features of the structural change described earlier can be seen clearly: the elongation of droplets and the gradual tilt of the columns. It should be noted that although the structural anisotropy can be described by an ellipsoid, the real structure is not ellipsoidal except in the initial time stage. More detail can be seen in Fig. 7, where the lengths of the semiaxes and the tilt angle are plotted as a function of time. As expected in the absence of an electric field, all the lengths are almost the same. Immediately after application of the electric field, the $c$ axis increases while the $a$ and $b$ axes initially decrease at the same rate, indicating that the structure is axially symmetric (prolate spheroid). At around $1 \mathrm{~s}$, the degeneracy of $a$ and $b$ is lifted, giving rise to a general ellipsoid. In the meantime,

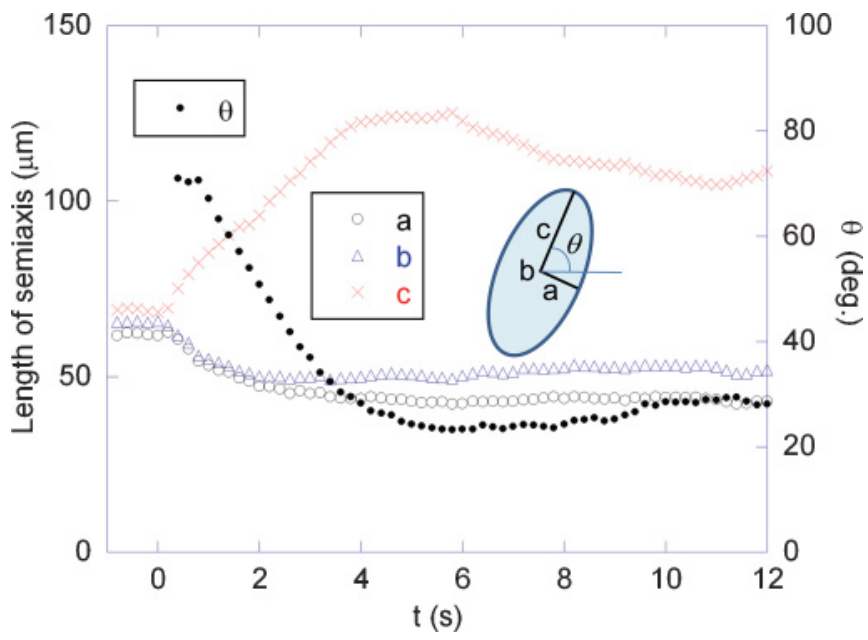

FIG. 7. (Color online) Time dependence of $a, b, c$, and $\theta$. 
the tilt angle $\theta$ gradually decreases. It should be noted that if the lengths of the semiaxes are constant, $q_{z x}$ should take a maximum at $\theta=45^{\circ}$, however, in our result $\theta$ reaches $45^{\circ}$ at $2.4 \mathrm{~s}$ (Fig. 7), whereas $q_{z x}$ peaks at about $3.1 \mathrm{~s}$ (Fig. 3). This is because $c$ is still increasing around $2.4 \mathrm{~s}$, as is seen from Fig. 7.

Now we turn our attention to the shear stress. For a blend of two Newtonian fluids with equal viscosity, the shear stress can be separated into three parts:

$$
\sigma=\sigma_{v}+\sigma_{\mathrm{if}}+\sigma_{e}
$$

where $\sigma_{v}, \sigma_{\text {if }}$, and $\sigma_{e}$ are the viscous stress, the interfacial stress, and the electric stress, respectively. The first two of these stresses can be expressed by the equations $\sigma_{v}=\eta \dot{\gamma}$ and $\sigma_{\text {if }}=-\Gamma q_{z x}$, where $\eta$ is the viscosity, $\dot{\gamma}$ is the shear rate, and $\Gamma$ is the interfacial tension. The expression for $\sigma_{e}$ will be derived later. The experimental separation is performed as follows. As can be seen from $\sigma_{v}=\eta \dot{\gamma}$, the viscous stress vanishes when the shear flow is stopped. Similarly, $\sigma_{e}$ should vanish when the electric field is removed, because $\sigma_{e}$ must come from the Maxwell stress tensor, $T_{i j}=\varepsilon E_{i} E_{j}-\varepsilon E^{2} \delta_{i j} / 2$, where $\varepsilon$ is the dielectric constant. Hence, we can devise a procedure for the separation of the three stresses.

During a measurement, after applying the step electric field under a steady shear flow, both the electric field and the shear flow are intermittently turned off and on within a short interval (duration: 0.24 s). The result is shown in Fig. 8(a), where the experimental conditions are the same as those shown in Fig. 3 except for the intermittent removal of the electric field and the shear flow. The details at around $6.8 \mathrm{~s}$ are shown in Fig. 8(b), where the time scale is expanded. First, the electric field is removed and the sudden decrease in shear stress corresponds to $\sigma_{e}$. Next, $0.06 \mathrm{~s}$ later, the shear flow is stopped to obtain $\sigma_{v}$. The remaining shear stress is assumed to correspond to $\sigma_{\text {if }}$. Lastly, both the shear flow and the electric field are applied again at an interval of $0.06 \mathrm{~s}$. The outline of the stress response in Fig. 8(a) is comparable to that in Fig. 3 except for the sudden changes, implying that the intermittent removal of the electric field and shear flow should not affect the structural evolution. From the changes shown in Fig. 8, we can separately obtain the three stresses in Eq. (5).

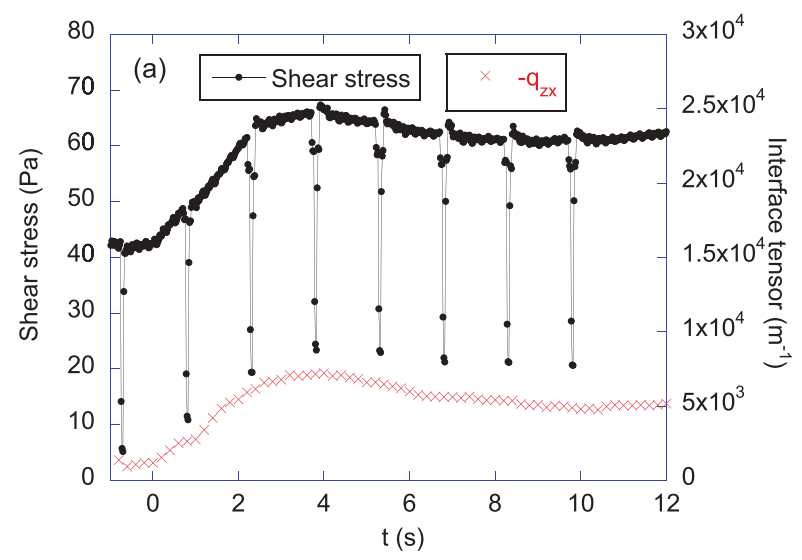

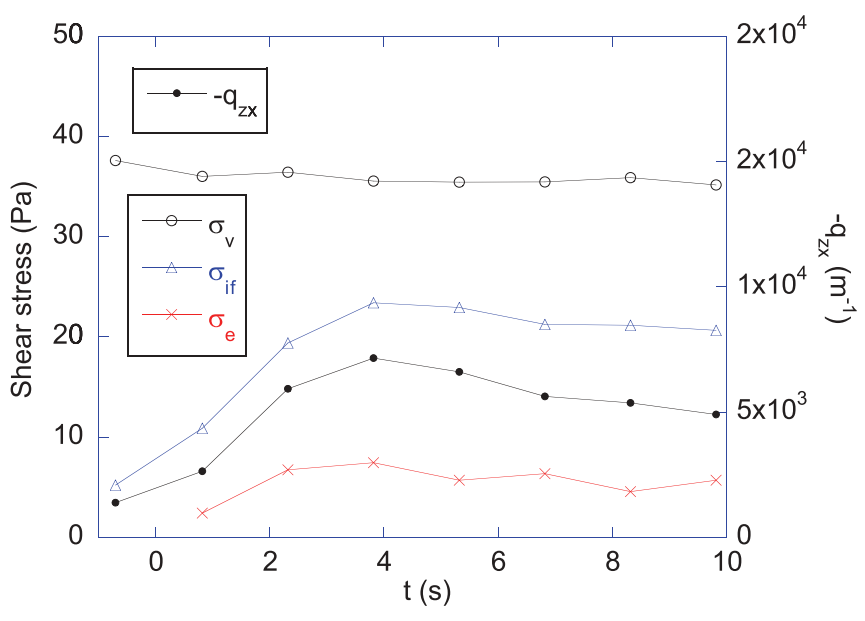

FIG. 9. (Color online) Time dependence of viscous stress $\sigma_{v}$, interfacial stress $\sigma_{\mathrm{if}}$, and electric stress $\sigma_{e}$, obtained from data shown in Fig. 8 .

Figure 9 shows the time dependence of the stresses, together with $-q_{z x}$. The viscous stress $\sigma_{v}$ is almost constant, as expected, giving a value of around $35 \mathrm{~Pa}$, which is in good agreement with the value $33.5 \mathrm{~Pa}$ calculated from $\eta=33.5 \mathrm{~Pa}$ s and $\dot{\gamma}=1 \mathrm{~s}^{-1}$. On the other hand, the interfacial stress $\sigma_{\mathrm{if}}$ depends on time and its behavior is similar to that of $-q_{z x}$. Interestingly, the electric stress $\sigma_{e}$ also shows a similar time dependence as $-q_{z x}$. As shown in Fig. 10, both $\sigma_{\text {if }}$ and $\sigma_{e}$ appear to be proportional to $-q_{z x}$. As for $\sigma_{\text {if }}$, this result indicates the validity of $\sigma_{\text {if }}=-\Gamma q_{z x}$ and the slope of the straight line in Fig. 10 gives an interfacial tension $\Gamma$ of approximately $-3.6 \times 10^{-3} \mathrm{~N} \mathrm{~m}^{-1}$, which is comparable with the value of $2.5 \times 10^{-3} \mathrm{~N} \mathrm{~m}^{-1}$ determined by the deformed drop retraction method [23].

The proportional relation between $\sigma_{e}$ and $-q_{z x}$ can be explained by supposing that there are ellipsoidal droplets dispersed in electric and shear flow fields having the same size and shape, and that the hydrodynamic interactions between them are negligible. In a steady state, the balance between the hydrodynamic and electric torques on each ellipsoid yields

$$
\sigma_{e}=-K_{y}^{(e)} \phi / v
$$

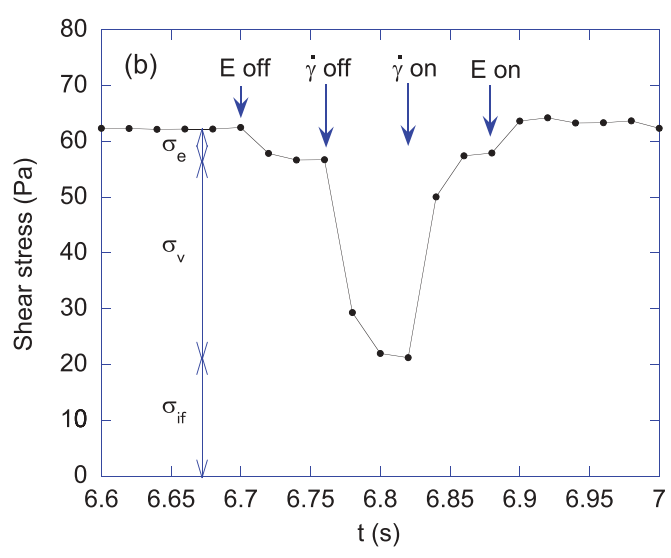

FIG. 8. (Color online) (a) Time dependence of the shear stress when the electric field and the shear flow are removed intermittently and (b) a part expanded between 6.6 and $7 \mathrm{~s}$. 


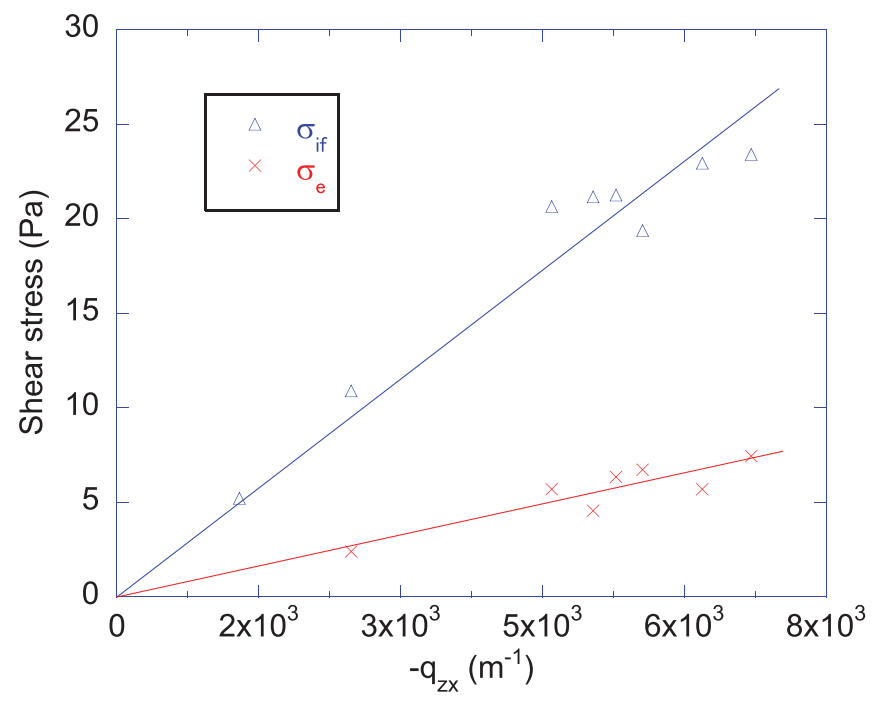

FIG. 10. (Color online) Dependence of $\sigma_{\text {if }}$ and $\sigma_{e}$ on $-q_{z x}$.

where $K_{y}^{(e)}$ is the $y$ component of the electric torque exerted on each ellipsoid, $\phi$ is the volume fraction of droplets, and $v$ is the volume of a droplet [24]. Strictly speaking, the system under consideration here is not in a steady state, but Eq. (6) holds approximately if the structure changes slowly. The electric torque $K_{y}^{(e)}$ is written as

$$
K_{y}^{(e)}=-p_{x} E
$$

where $p_{x}$ is the $x$ component of the induced dipole moment and $E$ is the electric field applied along the $z$ direction. The dipole moment $p_{x}$ is easily calculated as follows [25]:

$$
p_{x}=\frac{v \varepsilon_{2}\left(\varepsilon_{1}-\varepsilon_{2}\right)^{2}\left(n^{(a)}-n^{(c)}\right) \sin \theta \cos \theta E}{\left\{\varepsilon_{2}+\left(\varepsilon_{1}-\varepsilon_{2}\right) n^{(a)}\right\}\left\{\varepsilon_{2}+\left(\varepsilon_{1}-\varepsilon_{2}\right) n^{(c)}\right\}} .
$$

Here $\varepsilon_{1}$ and $\varepsilon_{2}$ are the dielectric constants of the LCP and PIB, respectively, and $n^{(a)}$ and $n^{(c)}$ are the depolarization factors defined as

$$
\begin{aligned}
& n^{(a)}=\frac{a b c}{2} \int_{0}^{\infty} \frac{d s}{\left(s+a^{2}\right) \sqrt{\left(s+a^{2}\right)\left(s+b^{2}\right)\left(s+c^{2}\right)}} \\
& n^{(c)}=\frac{a b c}{2} \int_{0}^{\infty} \frac{d s}{\left(s+c^{2}\right) \sqrt{\left(s+a^{2}\right)\left(s+b^{2}\right)\left(s+c^{2}\right)}}
\end{aligned}
$$

$n^{(b)}$ is similarly defined. The depolarization factors can be related to the surface tensor in the principal axis frame as follows. We have similar equations for $n^{(\alpha)}$ and $Q_{\alpha \alpha}: n^{(a)}+$ $n^{(b)}+n^{(c)}=1$ and $Q_{a a} / Q+Q_{b b} / Q+Q_{c c} / Q=1$ (note that $Q_{\alpha \alpha} / Q=\left\langle n_{\alpha}^{2}\right\rangle$, where $\langle\cdots\rangle$ denotes the average). Furthermore, for a sphere $n^{(a)}=n^{(b)}=n^{(c)}=1 / 3$ and $Q_{a a} / Q=$ $Q_{b b} / Q=Q_{c c} / Q=1 / 3$, and for a cylinder $n^{(a)}=n^{(b)}=1 / 2$, $n^{(c)}=0$ (the unique axis is taken along the $c$ axis) and $Q_{a a} / Q=Q_{b b} / Q=1 / 2, Q_{c c} / Q=0$, implying that the depolarization factors may be approximated by $n^{(\alpha)} \simeq Q_{\alpha \alpha} / Q=$ $\left\langle n_{\alpha}^{2}\right\rangle$, which has been numerically proven. By using this

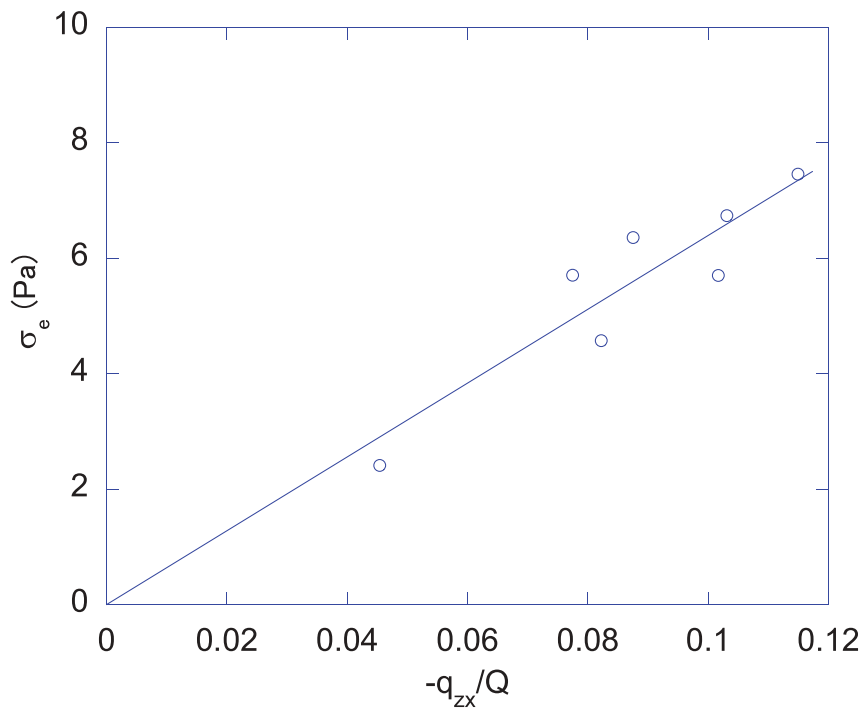

FIG. 11. (Color online) Plot of $\sigma_{e}$ vs $-q_{z x} / Q\left(=-\left\langle n_{z} n_{x}\right\rangle\right)$.

approximation and assuming that the ellipsoid is nearly spherical, the electric stress is given by the following:

$$
\sigma_{e}=\frac{9 \varepsilon_{2}\left(\varepsilon_{1}-\varepsilon_{2}\right)^{2}}{\left(\varepsilon_{1}+2 \varepsilon_{2}\right)^{2}} E^{2} \phi\left(-q_{z x} / Q\right),
$$

where the relation $\left(Q_{a a}-Q_{c c}\right) / Q \sin \theta \cos \theta=-q_{z x} / Q(=$ $\left.-\left\langle n_{z} n_{x}\right\rangle\right)$ is used.

The electric stress is plotted as a function of $-q_{z x} / Q$ in Fig. 11. The slope is about $64 \mathrm{~Pa}$, comparable with $156 \mathrm{~Pa}$ obtained from Eq. (10). With $Q=S / V$, where $S$ is the total interface area, Eq. (10) can be rewritten as

$$
\sigma_{e}=-\Gamma_{e} q_{z x}
$$

with

$$
\Gamma_{e}=\frac{9 \varepsilon_{2}\left(\varepsilon_{1}-\varepsilon_{2}\right)^{2}}{\left(\varepsilon_{1}+2 \varepsilon_{2}\right)^{2}} E^{2} V_{d} / S,
$$

where $V_{d}$ is the total volume of droplets. Equation (11) corresponds to $\sigma_{\text {if }}=-\Gamma q_{z x}$ for the interfacial stress. Therefore,

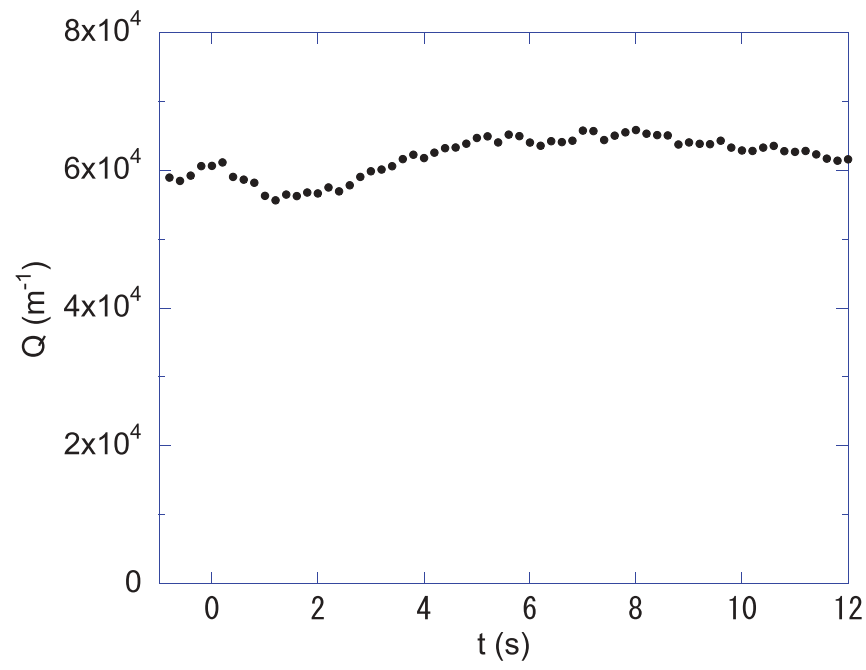

FIG. 12. (Color online) Time dependence of the interface area density $Q$. 


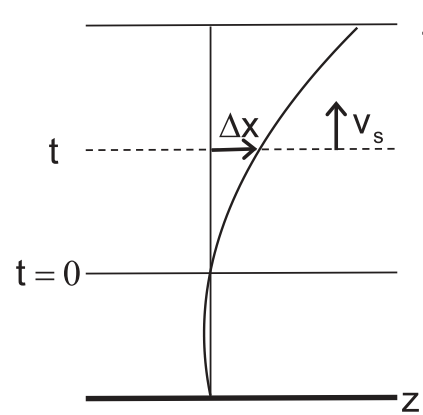

(a)

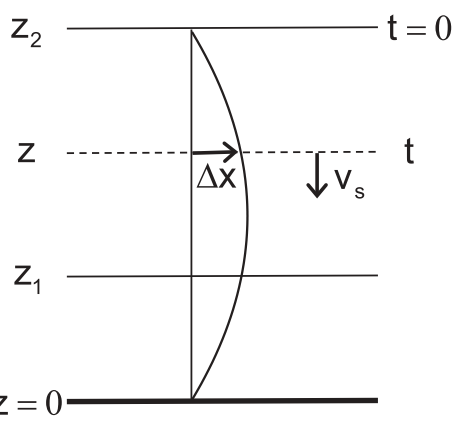

(b)
FIG. 13. (a) Upward scan and (b) downward scan, where the focal plane is located at $z$ and $v_{s}$ is the scan speed.

$\Gamma_{e}$ in Eq. (12) can be regarded as the electric interfacial tension. However, it should be noted that $\Gamma_{e}$, unlike $\Gamma$, is not always constant because $S$ can change. As shown in Fig. 12, $Q=S / V$ is almost independent of time, indicating that $\Gamma_{e}$ can be regarded as constant in our system. For this reason, $\sigma_{e}$ is proportional to $q_{z x}$ in Fig. 10. In closing, we should emphasize that Eq. (11) is theoretically derived for an ellipsoid-dispersed structure, but may be equally valid for a network structure, as has been shown experimentally.

\section{CONCLUSIONS}

Three-dimensional images were obtained in the transient process in which a polymer blend, consisting of two polymers with equal viscosity, was subjected to a step electric field under steady shear flow, and stress was measured simultaneously. In addition, an experiment to separate the viscous, interfacial, and electric stresses from the total shear stress by intermittently removing the electric field and shear flow was performed. The time dependence of the interface tensor was calculated from the images, and the contribution of the interfacial tension was confirmed to be expressed as $-\Gamma q_{z x}$. Furthermore, the electric stress was found to be proportional to a dimensionless quantity $q_{z x} / Q=\left\langle n_{z} n_{x}\right\rangle$, and the proportionality constant was theoretically estimated. Thus, we have successfully determined the relationship between the structure (the interface tensor) and the shear stress in the transient process. However, the mechanism of network formation under shear flow and electric fields remains unclear, and therefore should be studied based on evolution equations such as the Doi-Ohta model [2].

\section{ACKNOWLEDGMENTS}

We would like to thank Takao Ohta, Takahiro Sakaue, and Kosuke Yakubo for valuable discussions. This work was supported by a Grant-in-Aid for Scientific Research [Priority Area "Soft Matter Physics" (Contract No. 463), C (Contract No. 22540416), and Wakate B (Contract No. 21740306).

\section{APPENDIX: CORRECTION FOR 3D IMAGES}

The following correction was made to the captured images. The focal plane is scanned between $z=z_{1}$ and $z_{2}$, where $z_{2}>z_{1}$, and the glass surface is located at $z=0$, as shown in Fig. 13. First, let us consider the upward scan from $z_{1}$ to $z_{2}$ [Fig. 13(a)]. It is assumed that the focal plane is at $z=z_{1}$ at $t=0$. At time $t$, the focal plane is at $z=z_{1}+v_{s} t$, where $v_{s}$ is the scan speed or the vertical speed of the focal plane. As the flow velocity at $z$ is $\dot{\gamma} z$, the horizontal displacement is given as $\Delta x=\dot{\gamma} z t=\dot{\gamma} / v_{s} z\left(z-z_{1}\right)$. Thus, the 2D image at $z$ is moved back by $\Delta x$. It should be noted that this correction is most effective when all of the points in the blend move in accordance with the shear strain. For the downward scan [Fig. 13(b)], $\Delta x$ is given as $\dot{\gamma} / v_{s} z\left(z_{2}-z\right)$. It is clear that the correction for the downward scan is smaller than that for the upward one. As a result, the 3D images are constructed from the $2 \mathrm{D}$ images obtained by scanning downward.
[1] C. L. Tucker III and P. Moldenaers, Annu. Rev. Fluid Mech. 34, 177 (2002).

[2] M. Doi and T. Ohta, J. Chem. Phys. 95, 1242 (1991).

[3] G. K. Batchelor, J. Fluid Mech. 41, 545 (1970).

[4] A. Onuki, Phys. Rev. A 35, 5149 (1987).

[5] A. Onuki, Europhys. Lett. 28, 175 (1994).

[6] M. Takahashi, P. H. P. Macaubas, K. Okamoto, H. Jinnai, and Y. Nishikawa, Polymer 48, 2371 (2007).

[7] J. Mellema and W. M. Willemse, Physica A 122, 286 (1983).

[8] K. Okamoto and M. Takahashi, Nihon Reoroji Gakkaishi 36, 43 (2008).

[9] K. Tajiri, K. Ohta, T. Nagaya, H. Orihara, Y. Ishibashi, M. Doi, and A. Inoue, J. Rheol. 41, 335 (1997).

[10] A. Inoue, Y. Ide, and H. Oda, J. Appl. Polym. Sci. 64, 1319 (1997).

[11] H. Kimura, K. Aikawa, Y. Masubuchi, J. Takimoto, K. Koyama, and K. Minagawa, Rheol. Acta 37, 54 (1998).
[12] X. Pan and G. McKinley, J. Colloid Interface Sci. 195, 101 (1997).

[13] J. Ha and S. Yang, J. Rheol. 44, 235 (2000).

[14] H. Orihara, Y. Ikeyama, S. Ujiie, and A. Inoue, J. Rheol. 47, 1299 (2003).

[15] H. Orihara, T. Shibuya, T. Nagaya, and S. Ujiie, J. Phys. Soc. Jpn. 75, 063802 (2006).

[16] Y. H. Na, K. Aida, R. Sakai, T. Kakuchi, and H. Orihara, Phys. Rev. E 80, 061803 (2009).

[17] H. Orihara, K. Tanabu, and Y. H. Na, J. Phys. Soc. Jpn. 77, 093802 (2008).

[18] H. Orihara, Y. Nishimoto, K. Aida, Y. H. Na, T. Nagaya, and S. Ujiie (accepted for publication in J. Phys. Condens. Matter).

[19] H. Orihara, A. Taki, M. Doi, and A. Inoue, J. Rheol. 45, 1479 (2001).

[20] K. Aida, Y. H. Na, T. Nagaya, and H. Orihara, Phys. Rev. E 80, 041807 (2009). 
[21] See supplementary material at [http://link.aps.org/ supplemental/10.1103/PhysRevE.83.026302]. The movie of the structure is played at real time speed from $t=-1$ to $12 \mathrm{~s}$.

[22] See supplementary material at [http://link.aps.org/ supplemental/10.1103/PhysRevE.83.026302]. The movie of the ellipsoid is played at real time speed from $t=-1$ to $12 \mathrm{~s}$.
[23] A. Luciani, M. F. Champagne, and L. A. Utracki, J. Polym. Sci. B 35, 1393 (1997).

[24] T. C. Halsey, J. E. Martin, and D. Adolf, Phys. Rev. Lett. 68, 1519 (1992).

[25] L. D. Landau, E. M. Lifshitz, and L. P. Pitaevskii, Electrodynamics of Continuous Media, 2nd ed. (Pergamon, Oxford, 1984). 